\title{
Early Bilateral Sensory Deprivation Blocks the Development of Coincident Discharge in Rat Barrel Cortex
}

\author{
Ayan Ghoshal, Pierre Pouget, Maria Popescu, and Ford Ebner \\ Department of Psychology, Center for Integrative and Cognitive Neuroscience, Vanderbilt Vision Research Center, Vanderbilt University, Nashville, \\ Tennessee 37240
}

\begin{abstract}
Several theories have proposed a functional role for synchronous neuronal firing in generating the neural code of a sensory perception. Synchronous neural activity develops during a critical postnatal period of cortical maturation, and severely reducing neural activity in a sensory pathway during this period could interfere with the development of coincident discharge among cortical neurons. Loss of such synchrony could provide a fundamental mechanism for the degradation of acuity shown in behavioral studies. We tested the hypothesis that synchronous discharge of barrel cortex neurons would fail to develop after sensory deprivation produced by bilateral whisker trimming from birth to postnatal day 60 . By studying the correlated discharge of cortical neuron pairs, we found evidence for strong correlated firing in control animals, and this synchrony was almost absent among pairs of cortical barrel neurons in deprived animals. The degree of synchrony impairment was different in subregions of rat barrel cortex. The model that best fits the data is that cortical neurons receiving direct inputs from the primary sensory (lemniscal) pathway show the greatest decrement in synchrony following sensory deprivation, while neurons with diverse inputs from other areas of thalamus and cortex are relatively less affected in this dimension of cortical function.
\end{abstract}

Key words: barrel cortex; sensory deprivation; coincident discharge; JPSTH; rats; septum

\section{Introduction}

The neural code of a sensory experience is the result of ensemble neural activity that contains all of the information needed to generate a perception. The code can be based on instantaneous firing rates, integrated firing rates, mean interspike interval duration of a group of specialized neurons, or a cluster of neurons having correlated responses (Eggermont, 2001, 2006, 2007). Thus, the correlated spiking activity within a neural assembly can improve the accuracy of the neural code (Miller, 1996; Abbott and Dayan, 1999; Rolls et al., 2003).

Sensory information is used continuously by animals when they explore their environment, and correlated neuronal discharge in the sensory pathway is one important mechanism for adapting to new stimuli in the environment. Detecting correlation allows an animal to make inferences about the environment. For example, Engel et al. (1991) showed that neurons in visual cortex exhibit correlated spiking activity when they have similar orientation sensitivity, and such cells are found over large extents of VI cortex. Examples of significant correlated neural discharge exist in the auditory system, such as those produced by binaural interactions (Brosch and Schreiner, 1999), and in the second so-

Received Sept. 16, 2008; revised Dec. 10, 2008; accepted Jan. 15, 2009.

This work was supported by National Institutes of Health Grant NS 25907. We acknowledge Drs. Mathew Diamond and David Kleinfeld for very helpful comments that improved the final manuscript.

Correspondence should be addressed to Dr. Ford Ebner, Department of Psychology, Wilson Hall, 11121st Avenue South, Vanderbilt University, Nashville, TN 37240. E-mail: ford.ebner@vanderbilt.edu.

D01:10.1523/JNEUROSCI.4427-08.2009

Copyright $\odot 2009$ Society for Neuroscience $\quad$ 0270-6474/09/292384-09\$15.00/0 matic sensory area (S-II) as a correlate of attention (Roy et al., 2007).

Sensory deprivation in rats beginning near birth has been shown to degrade the function of the cerebral cortex, and the loss persists even after normal activity levels are restored later in life. Normal kittens raised in complete darkness for 4-6 months appear behaviorally blind when they start using vision as adults, and they improve only marginally over time (Wiesel and Hubel, 1965). One carefully defined deficit produced by visual sensory deprivation in cats is a loss of normal acuity when tested by lines and gratings (Mitchell, 1988). In the rat whisker system, sensory deprivation degrades their ability to discriminate between stimuli of different spatial roughness, and this loss of acuity also persists for prolonged periods (Carvell and Simons, 1996). The deficits in acuity coupled with the requirement for correlated discharge in neural coding lead to the hypothesis that the maturation of network assemblies requires activity-based correlated discharge to develop normal function in cortical neuronal assemblies. If so, sensory deprivation produced by trimming rat whiskers would be predicted to interfere with the development of coincident neuronal firing in whisker-recipient barrel cortex. Although the cortical barrel looks structurally normal following bilateral whisker trimming from birth to maturity, the perturbations in neural coding and the consequent behavioral deficits might be accounted for by the failure of barrel neurons to develop appropriate correlated firing. We tested this hypothesis and found that sensory deprivation strongly interferes with the development of correlated discharge between neuronal pairs in barrel cortex. Thus, in this paper instead of focusing on the various characteristic details of correlation in barrel cortex, we have tried to iden- 
tify and characterize the differences in the degree of correlations between normal and sensory-deprived rats in barrels and septa.

\section{Materials and Methods}

All of the experiments performed for this report were approved by the Vanderbilt University Animal Care Committee (Institutional Animal Care and Use Committee), and were in accordance with the guidelines of the National Institutes of Health and the Society for Neuroscience in an Association for Assessment and Accreditation of Laboratory Animal Care-approved animal facility.

Sensory deprivation. Long-Evans rats (250-350 g, 2-3 months old) from four litters (eight animals total) were used for this study. Of these, four had all their whiskers trimmed on both sides of the face (bilaterally deprived group or BD) and four animals were handled but not trimmed (control or CON group). Whiskers were trimmed to the level of the fur for at least $60 \mathrm{~d}$ beginning at birth: the first $30 \mathrm{~d}$ (when the whisker growth rate was faster) the whiskers were trimmed every day and after postnatal day 30 the whiskers were trimmed every other day. During preweaning whisker trimming, the whisker-trimmed BD pups were caged with their CON littermates and nursed by their dam. After weaning, same-sex animals were housed in groups of three to five animals depending on their size. Five days before recording sessions the whiskers were allowed to regrow to a length sufficient for stimulation without moving the fur.

Surgery and recording. The rats were anesthetized with urethane (1.5 $\mathrm{g} / \mathrm{kg}, 30 \%$ aqueous solution, i.p.), and then mounted in a head holder that allowed free access to the whiskers (Narashige). Urethane is a general anesthetic that has been shown to have little effect on glutamate or GABAergic currents, but blocks Ba ${ }^{+}$-sensitive $\mathrm{K}^{+}$channels (Sceniak and Maciver, 2006). A craniotomy was made from 4 to $7 \mathrm{~mm}$ lateral to the midline and from 0 to $5 \mathrm{~mm}$ posterior to Bregma to expose the left barrel cortex. Body temperature was maintained at $37^{\circ} \mathrm{C}$ with a feedback actuated heating pad (Harvard Apparatus). Supplementary injections (10\% of the initial dose) were given as needed to maintain the anesthesia at stage III-3 (Friedberg et al., 1999). After making a small opening in the dura mater microelectrodes were advanced in columnar penetrations perpendicular to the cortical surface. Contact with the pia was identified visually through an operation microscope. We used three quartz glass insulated, platinum/iridium microelectrodes having 2-6 $\mathrm{M} \Omega$ resistance (Thomas Recording). The electrodes were separated by $305 \mu \mathrm{m}$ and advanced into the brain independently using an Eckhorn microdrive system (Thomas Recordings). The analog waveforms from the Thomas system were transferred to a Plexon MAP system where they were digitized at $40 \mathrm{kHz}$. Multiunit activity was viewed online using Sort Client software (Plexon), and stored for off-line analysis. The electrodes were first advanced to layer IV where receptive fields were mapped manually, and the three whiskers that evoked the largest amplitude responses were identified for each electrode based on multiunit poststimulus time histograms (PSTHs) constructed online in PeriEvent Client (Plexon). Multiunit activity was recorded from layer IV $(450-800 \mu \mathrm{m})$ ( Li et al., 2005). The recording depth was measured on-line by Eckhorn microdrive readings and later correlated with histological reconstructions of the electrode tracks and microlesions. Electrodes were advanced or retracted in $100 \mu \mathrm{m}$ vertical increments to reduce the probability of recording twice from the same unit. Moreover, recording was discontinued when spindling activity in the cortex was observed and resumed only after spindling had stopped.

Whisker stimulation. After the regrowth period, the whiskers in both $\mathrm{CON}$ and $\mathrm{BD}$ animals were trimmed to $5 \mathrm{~mm}$ beyond the fur to keep stimulus distance equivalent. A piezoelectric stimulator was used to deliver 100 stimuli in a caudal direction at $0.5 \mathrm{~Hz}(600 \mu \mathrm{m}$ amplitude, $4 \mathrm{~ms}$ duration, $2 \mathrm{~ms}$ rise time with custom rounded peak). The piezoelectric wafer was actuated by a "custom" waveform programmed in a digital stimulator (DS8000 WPI) that in turn was controlled by a Spike 2 (CED) script program.

Histology. At the end of each recording session the electrodes were moved to $700 \mu \mathrm{m}$ below the pial surface and electrolytic lesions were made using a DC current of $1 \mu \mathrm{A}$ for $2 \mathrm{~min}$. This current level produces a clearly visible lesion in cytochrome oxidase-stained tangential slices through layer IV, and can be used to localize the electrodes in barrel or septum columns without destroying the electrodes. At the end of each experiment the animals were given a lethal overdose of urethane and perfused transcardially with PBS followed by phosphate-buffered $4 \%$ paraformaldehyde. Brains were postfixed overnight and saturated in $30 \%$ sucrose. The cortex was flattened, sectioned tangentially, and stained for $\mathrm{CO}$ activity to assign penetrations and lesion sites to barrels or septa.

Data analysis. Off-line cell sorting of single units was performed using Offline Sorter (Plexon). Typically 2-3 units per electrode could be selected for further analysis. Only cells well separated as three-dimensional clusters and with qualitatively distinctly different waveform shapes (see supplemental Fig. $2 A, B$, available at www.jneurosci.org as supplemental material) were included for further analysis. The magnitude of response was defined as the total number of spikes in the $100 \mathrm{~ms}$ following the stimulus and was calculated for all stimulated whiskers for each cell. There was no threshold set for the level of response to be included. We then determined the principal whisker (PW) as being the whisker eliciting the highest magnitude of response for barrel cells. For septal cells, responses to all whiskers stimulated were included. PSTHs were constructed for each cell in response to stimulation of the principal whisker using a custom NEX script (provided by Dr. Alexander Kirillov, NEX Technologies, Littleton, MA) and custom software (The MathWorks).

Spike time synchrony. There are multiple time scales in which temporal correlation can be examined. To assess precise spike synchrony, small time bins ( $1-5 \mathrm{~ms})$ often have been used; however, such small bins require relatively huge spike counts to be valid. Coarse temporal correlation is considered at time scales $>20 \mathrm{~ms}$. Bin sizes between 1 and $15 \mathrm{~ms}$ are commonly reported (e.g., in S1: Aertsen et al., 1989; Hsiao et al., 1993; Roy et al., 2007). In the present study, spike correlation was examined over $2000 \mathrm{~ms}$ windows divided into 200 bins so that $10 \mathrm{~ms}$ bins would still include the response transient peak. Ten millisecond bins allowed us to analyze coincident discharge over time especially for the period of relatively low firing during sustained "spontaneous" activity. The correlation coefficients were extracted by subtracting the mean values of number of spikes for each cell: $\delta n_{i}^{(k)}(\mu) \equiv n_{i}^{(k)}(\mu)-\left\langle n_{i}(\mu)\right\rangle$ and $\delta n_{j}^{(k)}(\nu) \equiv$ $n_{j}^{(k)}(\nu)-\left\langle n_{j}(\nu)\right\rangle$, respectively, where $n_{i}^{(k)}(\mu)$ and $n_{j}^{(k)}(\nu)$ are the numbers of spikes for each time bin across each trial $k$ of each cell $(\mu$ and $\nu)$, and where $\left\langle n_{i}(\mu)\right\rangle$ and $\left\langle n_{j}(\nu)\right\rangle$ are the mean values of spikes at each bin ( $i$ and $\left.j\right)$ for each cell ( $\mu$ and $\nu$ ), so that the unconnected part of the correlation is defined as follows: $\left.\left\langle\delta n_{i}(\mu) \delta n_{j}(\nu)\right\rangle \equiv\left\langle n_{i}(\mu) n_{j}(\nu)\right\rangle-\left\langle n_{i}(\mu)\right\rangle n_{j}(\nu)\right\rangle$.

When normalized, this value becomes a coefficient of correlation, which was renamed as the joint poststimulus time histogram (JPSTH) by Gerstein and colleagues, having values ranging from -1 to +1 (Gerstein et al., 1989).

The JPSTH matrix represents earlier time in the lower left corner and later time in the upper right; the bins along this main diagonal measure the coincidences with 0 time lag (see supplemental Fig. $2 C$, available at www.jneurosci.org as supplemental material). The JPSTH can be summarized in two additional plots. First, the time-averaged crosscorrelogram is computed by summing the JPSTH bins parallel to the main diagonal; the cross-correlogram measures the average positive or negative correlation across the entire interval of analysis. Second, the coincidence histogram represents the coincident or near-coincident firing of the cells time locked to values in each PSTH. Although the bin-bybin dynamics of the correlation are not analyzed here, examination of the JPSTH revealed that the correlated spike times occur during the sustained portion of the stimulus-driven activity, more than at the transient responses to stimulus onset and removal. The spike timing coincidence will be examined elsewhere. To test the reliability of the normalized JPSTH, we repeated the JPSTH calculation on a set of spike trains in which no correlation should be present but the spike train variability is preserved. This shuffled JPSTH was generated by shuffling the order of the trials for one of each pair of neurons. It is important to emphasize that the trial-by-trial variability present in the normalized JPSTH is preserved within the shuffled JPSTH even if no correlation should be observed. Only pairs showing no correlation for the shuffled JPSTH were considered for further analysis. Second, a simulated JPSTH was performed on a series of trials with spike trains derived from a nonhomogeneous Poisson process with the rate derived from the actual average spike density func- 
tion. Specifically, for each $1 \mathrm{~ms}$ interval we drew a random sample from the uniform distribution $[0,1]$. If, and only if, the sample was less than the normalized average spike density function value at that interval, would a simulated spike be counted. The simulated JPSTH was calculated from 50 simulated trials generated from the average spike density function for each pair of neurons. The two previous procedures allowed us to discard inappropriate normalizations.

Statistical analysis. The nonparametric Wilcoxon rank-sum test was used to determine significant differences $(p<0.05)$ between the cumulative distributions of peak-correlation coefficients. Paired one-tailed Student's $t$ test was performed to find the significance of the normalized correlation coefficients over simulated and shuffled coefficients and to analyze the difference between the peak-correlation coefficients during the stimulus-driven and the spontaneously active response epoch (see supplemental Discussion, available at www.jneurosci.org, for details on methods).

\section{Results \\ Calculating correlated discharge in barrel cortex neuronal pairs}

JPSTHs were constructed as described to show the occurrence of neuronal synchrony over time after a stimulus and to determine the average peak-correlation values between pairs of neurons in control (CON, $n=4)$ and bilaterally whiskertrimmed (BD, $n=4)$ rats. The broad $2 \mathrm{~s}$ time scale was chosen for the JPSTH analysis to show coincidence of spikes both in the stimulus-driven short-latency epochs and in the long-latency (1000-2000 ms after stimulus) spontaneously active period as displayed in the coincidence histograms. For each animal group, the peak correlation observed between the neuronal pairs in the normalized JPSTH was identified as significant $(p<0.001)$ by comparing it with those obtained in the simulated and the shuffled JPSTH using the paired $t$ test. The correlation peaks observed in the cross-correlograms of neurons in barrel cortex were narrow and almost always had a time lag close to 0 . Moreover, the cross-correlograms did not show oscillations, but there were noticeable oscillations in the coincidence histograms for the neuronal pairs.

\section{Neuronal synchrony within a single barrel}

Spike synchrony was calculated in $78 \mathrm{CON}$ and $70 \mathrm{BD}$ pairs of barrel neurons. The JPSTH analysis showed that most of the neuronal pairs in CON barrels were highly correlated in their discharge. This spike synchrony was observed for the entire $2 \mathrm{~s}$ poststimulus period of recording after principal whisker (PW) stimulation. Fig. $1 \mathrm{~A}$ illustrates the typical correlated activity observed between two barrel neurons (color-coded waveforms for each cell in insert) in a CON barrel. The high degree of correlated
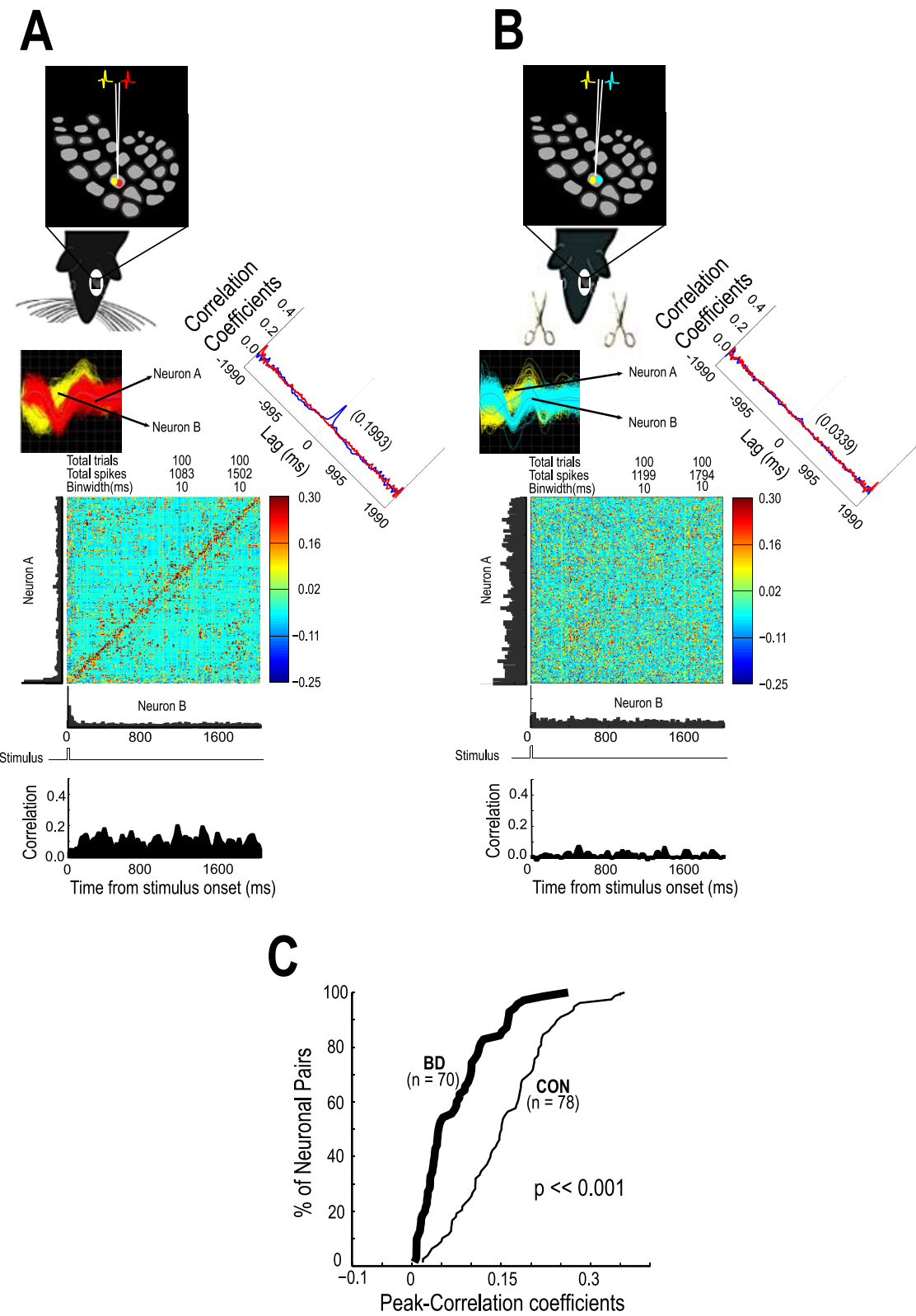

Figure 1. Correlated discharge of two neurons in the same barrel. $A$, JPSTH of a single pair of CON barrel neurons (neuron A and B waveforms in inset) showing the PSTHs for each cell after 100 trials. The color-coded JPSTH matrix of correlation values and the cross-correlogram (angled display) with a peak correlation coefficient of 0.1993 at 0 time lag show the degree of correlated discharge between this pair of neurons. The stimulus trace represents a $4 \mathrm{~ms}$ whisker stimulus beginning at time 0 , following which the two neurons were simultaneously recorded. The degree of correlation over time along the diagonal (coincidence histogram) is shown at the bottom of $A$. In the cross-correlogram the normalized trace (in blue) has been superimposed with the shuffled trace (in red). $\boldsymbol{B}$, Similar data from a pair of barrel neurons in a BD rat. Note that these cells had nearly the same total number of spikes as the control pair, but these cells show no clear peak in the cross-correlogram and a peak-correlation coefficient of 0.0339 . C, Cumulative distribution of peak correlation in all barrel cell pairs in CON ( $n=78$ pairs) and BD ( $n=70$ pairs) conditions. The CON pairs show significantly higher correlation than the BD cells (Wilcoxon nonparametric test; $p<0.001$ ). with a maximum value of 0.1993 centered near a lag of $0 \mathrm{~ms}$. As shown in the PSTHs in Figure $1 \mathrm{~A}$, the firing rate of these two neurons changes dramatically over time after the stimulation. Rapid changes in firing rate could affect the quantification of spike synchrony. Thus, it is important to note that high correlation was detected even when the firing rate of the two neurons were low and had returned to background levels (i.e., from $\sim 200$ to $2000 \mathrm{~ms}$ after the stimulus). 


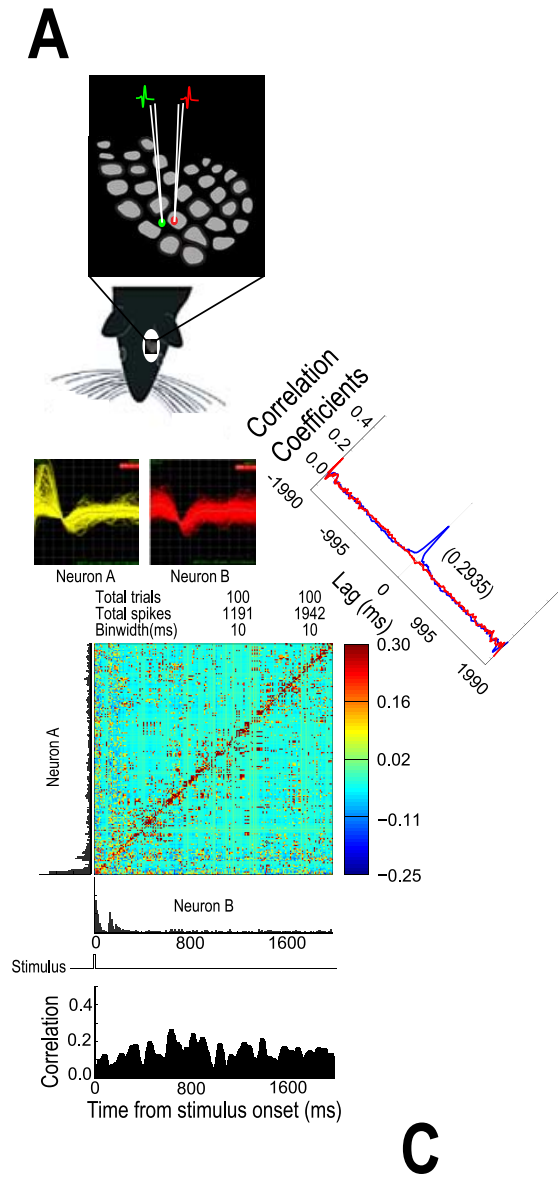

B

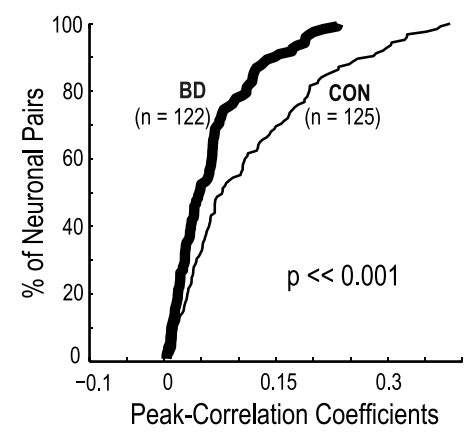

Figure 2. Correlated discharge of two neurons, one in a barrel and the other in a nearby septal location. $A, B$, Here the neurons are $\sim 300 \mu \mathrm{m}$ apart, with their firing rates recorded simultaneously from two adjacent electrodes following a $4 \mathrm{~ms}$ whisker stimulus represented by the stimulus trace. The waveforms are highly unlikely to be from the same cell even when the waveforms are similar in shape (waveforms displayed in separate inserts because they were recorded by 2 electrodes). Display similar to Figure 1. Here there is a detectable peak in the BD cross-correlogram (peak-correlation coefficient of 0.0785 ), but it is much reduced compared with CON pairs (peak-correlation coefficient of 0.2935 ). C, The difference is clearly seen when the population cumulative distributions of peak correlation coefficients of the $\mathrm{BD}(n=122)$ and $\operatorname{CON}(n=125)$ neuron pairs are compared with the Wilcoxon nonparametric test $(p<0.001)$.

When pairs of recorded neurons were located in a cortical barrel of a $\mathrm{BD}$ animal, correlated discharge could not be demonstrated. Figure $1 B$ illustrates this difference with an example of correlated discharge between a single pair of neurons in a "deprived" barrel. The cross-correlogram (average peak correlation coefficient of 0.0339 ) and the coincidence histogram show the low level of correlated discharge during the entire $2 \mathrm{~s}$ poststimulation period. An increase or reduction in the total number of spikes could also potentially affect the quantification of spike synchrony (Brody, 1999; Ventura et al., 2005). BD animals generally have been reported to show a low response magnitude (M. Popescu and F. Ebner, unpub- lished observations) (supplemental Fig. 1, available at www.jneurosci.org as supplemental material). However, the example in Figure $1 B$ shows two exceptional "deprived" neurons that produced a nearly identical number of spikes (to the same type and duration of stimulus as that of CON) compared with the CON example, but still failed to develop any synchrony. Thus, the diminution in the total number of spikes cannot be the sole determinant of reduced correlated discharge levels in deprived barrels.

Figure $1 C$ shows a cumulative distribution of peak correlation for the entire population of the CON and BD neuronal pairs in barrels. The CON distribution clearly has a higher range of peak-correlation coefficients than the "deprived" distribution within the barrel, and the values were significantly different $(p \ll 0.001$, Wilcoxon nonparametric test of cumulative distribution). Overall, there was a 53\% decrease of the mean peak correlation coefficients in $\mathrm{BD}$ neuronal pairs when compared with the CON group.

\section{Neuronal synchrony between barrels and septa}

Pairs of CON neurons $(n=125)$ and BD neurons $(n=122)$ were analyzed using two electrodes ( $\sim 300 \mu \mathrm{m}$ apart), one in a barrel and another in an adjacent region of the septum. The correlated discharge between pairs of barrel-septal neurons was high in CON animals. Figure $2 A$ shows a representative CON barrel-septal neuronal pair. The correlation peak was narrow and centered around a lag of $0 \mathrm{~ms}$. Again, even if the firing rate strongly changes over the course of a trial, the coincidence histogram shows high coincident spiking activity throughout the entire $2 \mathrm{~s}$ poststimulation period. On the other hand, the spike synchrony in BD barrel-septal pairs was again extremely low, as illustrated in Figure $2 B$, despite spike magnitudes (for this particular example) that were comparable with those of the CON example (Fig. 2A). The persistent lack of spike synchrony was revealed by the low average peak-correlation value (0.0785), and the coincidence histogram (Fig. 2B, bottom). Overall, the population cumulative distribution for the peak correlation of the BD group was significantly different (Fig. 2C) ( $p \ll 0.001$, Wilcoxon nonparametric test of cumulative distribution) when compared with the CON group. Moreover compared with the CON, the population mean peak correlation of the $\mathrm{BD}$ was reduced by $44 \%$ (Fig. $2 D$ ). Thus, $\mathrm{BD}$ had a strong negative impact on synchrony between cells in barrels and in the surrounding septa.

\section{Neuronal synchrony within the septa}

Correlated discharge between neurons in the septa was much less affected by BD than cells in other barrel cortex locations. Unlike 
the barrel and barrel-septum neuronal pairs, we observed that, not all the septal neuron pairs showed reduced synchrony in the BD animals. This result is illustrated in Figure 3, $A$ and $B$, with a typical example of a CON pair of septal neurons showing a peak-correlation coefficient of 0.1330 and a comparable BD septal pair with a similar peak-correlation coefficient of 0.1374 . Figure $3 C$ shows the cumulative distribution of the population peak correlations for the CON $(n=125)$ and $\operatorname{BD}(n=122)$ rats. The two distributions were statistically not different $(p>0.05$; Wilcoxon nonparametric test of cumulative distribution), but the plots reveal that there are an important percentage of neuronal pairs in the $\mathrm{BD}$ septa that are characterized by lower peak-correlation coefficients than CON (Fig. 3C, between arrows). The population peak correlation of the BD septa was on average reduced by $29 \%$ below CON.

To analyze the apparent multimodal cumulative distributions of the CON population peak-correlation coefficients (Fig. $3 C$ ), and the reduction of its mean value we examined the frequency and probability distributions of the peak correlations of the septal CON and BD populations (Fig. $4 A, B)$. As shown in Figure $4 A$, the frequency and probability distributions indicate that the CON septa have a range of peak-correlation coefficients varying from 0 to 0.4 , with only a subpopulation of cells showing relatively high (defined arbitrarily as $>0.15$ ) correlation (to the right of the red dashed line in Fig. $4 A$ ). Interestingly, in the BD distribution (Fig. $4 B$ ), the frequency of neuronal pairs with high correlation $(>0.15$ indicated by the red dashed line in Fig. $4 B$ ) was reduced by 49\% ( $p<0.01$; Wilcoxon nonparametric rank-sum test), but many neuronal pairs with relatively lower, but significant $(p<$ 0.01 ), correlation continued to be frequent in the $\mathrm{BD}$ septa suggesting that they were less affected by sensory deprivation. In other words, the effect of bilateral deprivation in reducing synchrony appears to be limited to a particular subpopulation of septal cells.

\section{Neuronal synchrony during stimulus-driven and spontaneous firing periods}

The pairs of PSTHs considered for the analyses showed a brief evoked response ( $\sim 0-50 \mathrm{~ms}$ epoch) following the short duration (4 ms) whisker stimulus, and a much longer ( 50-2000 ms) poststimulus epoch of background discharge. Since neuronal spike coincidence may provide an important platform for stimulus encoding, it is possible that the onset of a stimulus modifies the existing neuronal synchrony, rendering it different from the synchrony that occurs during the rest of the interstimulus interval. To test this idea we calculated the average coincidence of each

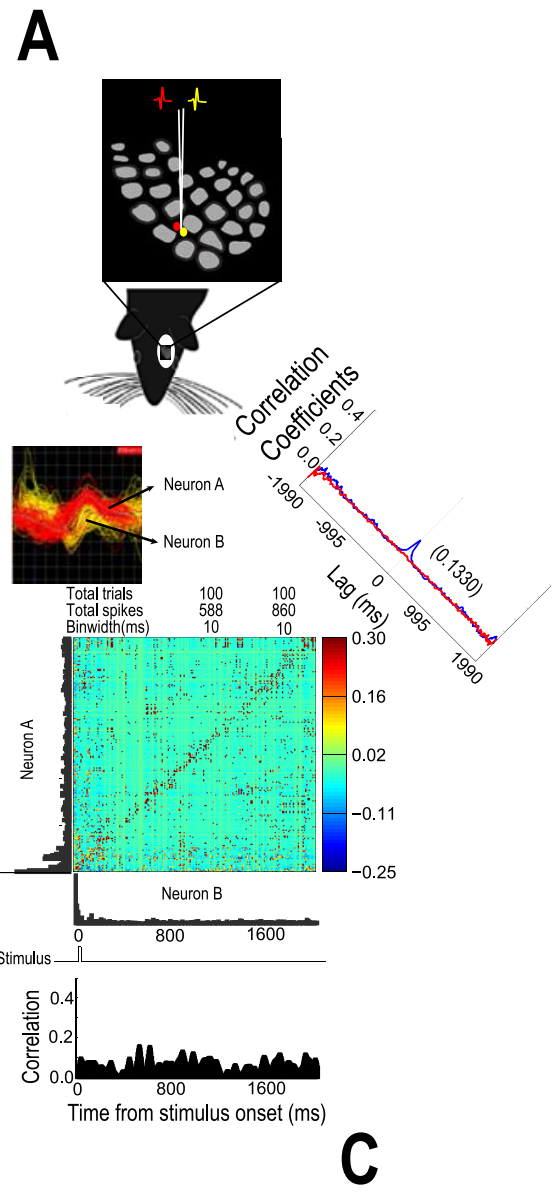

B
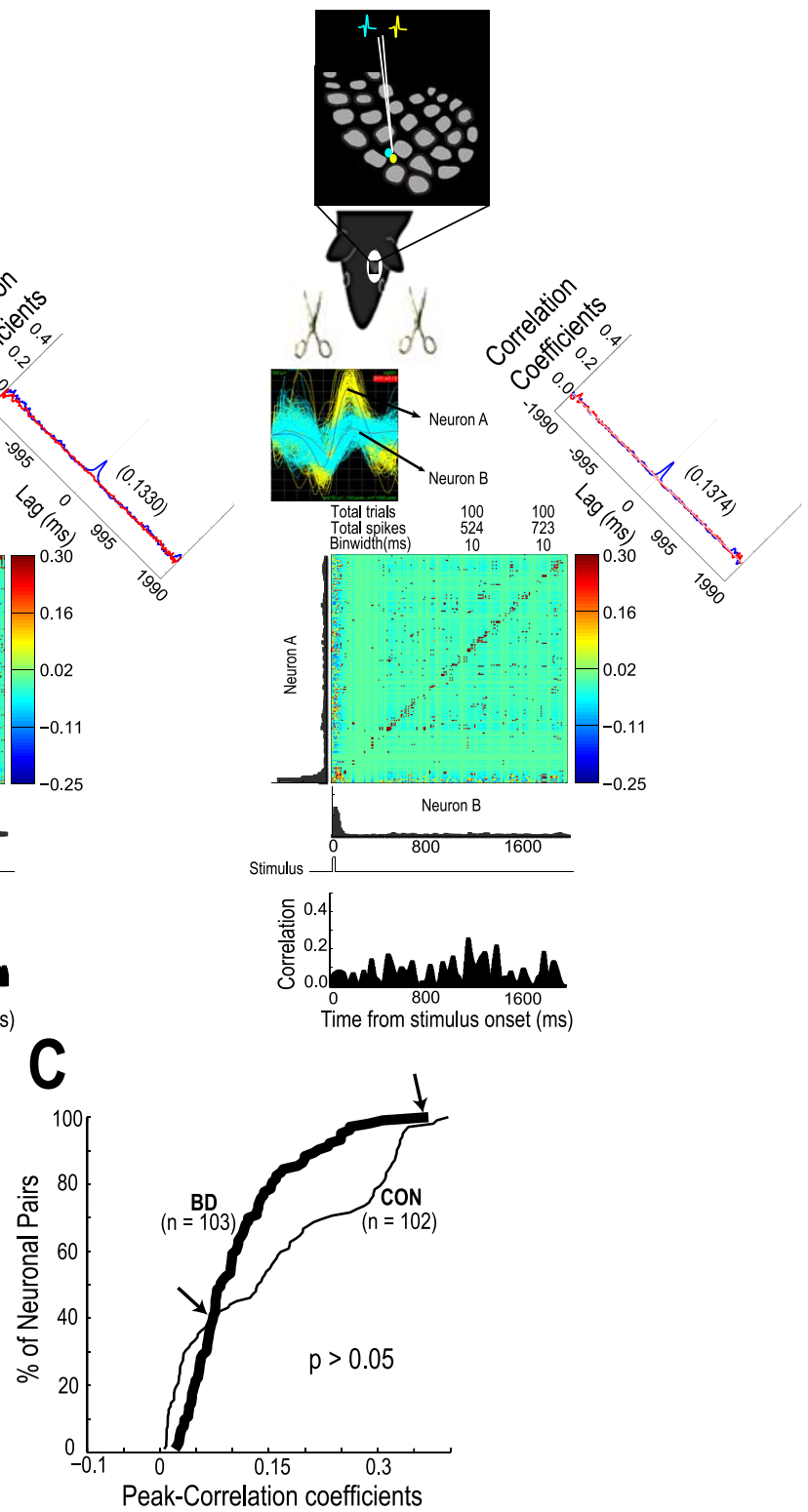

Figure 3. Correlated discharge of two neurons in the septal area between barrels. $\boldsymbol{A}, \boldsymbol{B}$, Display similar to Figure 1. In the septum, the degree of correlated discharge is less than in barrels and similar levels are maintained after $B D$ in these example cell pairs. C, Cumulative distribution of peak correlation coefficients in all septal cell pairs in CON ( $n=102$ pairs) and BD $(n=103$ pairs) conditions. Although the Wilcoxon nonparametric test shows no statistical difference $(p>0.05)$ between the two distributions, there is a noticeable percentage of $\mathrm{BD}$ neuron pairs (between arrows) characterized by lower peak-correlation coefficients than $\mathrm{CON}$. These were further analyzed in Figure 4.

neuronal pair during the stimulus-driven and the spontaneously active period. In the coincidence histogram, the "first" $50 \mathrm{~ms}$ (0-50 ms after stimulus) represented the time period containing almost all of the whisker stimulation-driven activity (Petersen and Diamond, 2000) and the "last" $50 \mathrm{~ms}$ (1950-2000 ms after stimulus or the $50 \mathrm{~ms}$ before the next stimulus) was assumed to be long enough after the stimulus to represent a spontaneous activity period for the neurons. The average coincidence during the first $50 \mathrm{~ms}$ after stimulus was calculated and compared with the average coincidence of the last $50 \mathrm{~ms}$ after stimulus using a Student's $t$ test for neuronal pairs in a group of barrel, septa, and barrel-septal pairs in both $\mathrm{CON}$ and $\mathrm{BD}$ rats (Fig. 5). In the CON animals the average coincidence of the last $50 \mathrm{~ms}$ was significantly 

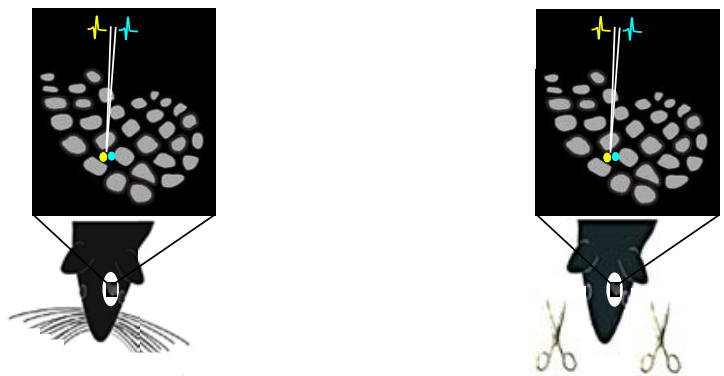

A

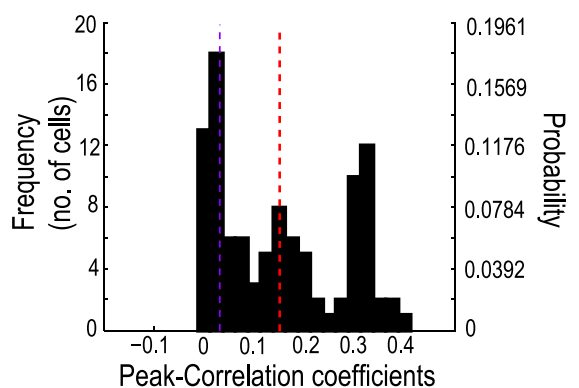

Figure 4. Frequency and probability distribution of peak correlations in pairs of septal neurons. The blue dashed line represents the upper value of confidence interval for a mean average of 0 ( $p<0.01$ ). Bin size $=0.025$. $A$, Frequency (number of cells) and probability distribution of $\mathrm{CON}$ peak-correlation coefficients in septum-septum neuronal pairs showing the range of peak correlations. The red dashed line has been arbitrarily placed at 0.15 to show a subpopulation of cells having relatively high peak correlation values (to the right of the dashed red lines). $\boldsymbol{B}$, Frequency and probability distribution of BD peak-correlation coefficients in septum-septum neuronal pairs. The red dashed line is placed at the same point in the $x$-axis of the distribution as the one in the CON case, to show the change in the distribution of cells. The low frequency of cell pairs to the right of the red dashed line reflects loss of the highly correlated neuronal pairs in the septum after $B D$. However, as seen to the left of the red dashed line, the septal neuronal pairs with low but significant peak-correlation coefficient values became more frequent after sensory deprivation.

higher than that of the first $50 \mathrm{~ms}$ for neuronal pairs in the barrel $(p<0.001)$ and septa $(p<0.01)$, and also for barrel-septal pairs $(p<0.001)$ (Fig. 5A-C, white bars). However, this difference was not significant after BD for within-barrel and barrel-septal neuronal pairs $(p>0.05)$ (Fig. $5 A, B$, gray bars). Interestingly, the difference between the average coincidences of the spontaneously active and the stimulus-driven firing period in the within-septa neuronal pairs were unaffected $(p<0.01)$ by bilateral sensory deprivation (Fig. 5C, gray bars). Similar results were produced when tested with the nonparametric rank-sum test. The temporal evolution of coincidence after the brief stimulus was also calculated as average coincidences in five successive epochs of $50 \mathrm{~ms}$ poststimulus time bins spaced regularly at $200 \mathrm{~ms}$ intervals during the $2000 \mathrm{~ms}$ after stimulus period (supplemental Fig. 3, available at www.jneurosci.org as supplemental material). However, further statistical analysis is required to completely characterize the pattern of the evolution of coincidence after stimulus.

\section{Discussion}

Sensory deprivation has been previously shown to have adverse effects on single-cell neurophysiology in sensory cortex (Wiesel and Hubel, 1965; Simons and Land, 1987; Rema et al., 2003; Shepherd et al., 2003; Shoykhet et al., 2005; Lee et al., 2007) (Popescu and Ebner, unpublished observations) (supplemental Fig. 1, available at www.jneurosci.org as supplemental material). The present study adds a new dimension to the effects of sensory deprivation by showing that neuronal ensemble functions are strongly degraded by sensory deprivation. The results show that sensory deprivation severely degrades coincident discharge in pairs of barrel-barrel and barrel- septum neurons in barrel cortex. The results confirm the presence of "synchrony at rest," that is, synchronous discharge of neurons during periods of "spontaneous" activity in this neural system (Swadlow et al., 1998; Zhang and Alloway, 2006). The correlation peaks were narrow in the cross-correlograms and almost always had near 0 lag. These results emphasize that barrel cells fire together coherently, and we conclude that sensory pathway activity during the first postnatal month is important for developing synchrony among barrel neurons.

The degree of change in synchronous discharge due to early sensory deprivation is complex and selective: when synchrony is almost obliterated between cell pairs in a barrel or barrel and septum, partial synchrony can be maintained by some neuron pairs in the septum. In control (CON) rats, the population of septal cell pairs shows a multimodal peak-correlation distribution which may reflect distinct subsets of cell pairs coupled by low, intermediate, and high levels of correlated discharge. In bilaterally whiskertrimmed animals, intermediate and highly synchronized neurons are severely affected, whereas low levels of correlated discharge develop among some septal cells. In addition to this overall dramatic loss of synchrony, the results show that the synchrony of the neurons in all parts of the barrel cortex is particularly reduced during the response to the stimulus (0-50 ms after stimulus). Interestingly, this relatively lower synchrony is not apparent in the "deprived" barrel and barrel-septal cell pairs, whereas the "deprived" septal cell pairs show a reduction of coincident firing during the stimulus-driven period similar to controls. Ongoing studies will determine whether this reduction in synchrony during the response to stimulation occurs in awake, attending animals.

One explanation for these results is that the abnormally low lemniscal activity coming into cortical barrels during the first month of life leads to a failure to develop correlated discharge among barrel neurons. A logical corollary is that perturbation of synchrony among barrel neurons in turn desynchronizes barrel to septum transmission, whereas connectional complexities and diversities in the different inputs and outputs of the septum (Kim and Ebner, 1999) permit it to preserve certain aspects of synchrony.

Behaviorally, a period of sensory deprivation similar to that used in the present study caused a loss of acuity in texture discrimination that persists even after normal activity levels are restored for months before and during behavioral training (Carvell and Simons, 1996). The deprived animals could distinguish between smooth and rough, suggesting that they were not cognitively impaired, but they could not distinguish between even grossly different rough surfaces that normally reared animals could distinguish at high performance levels. After normal rearing, synchronous spiking activity has been observed between almost all inhibitory barrel interneurons that receive potent monosynaptic input from the ventral posterior medial thalamic nucleus, but similar correlated discharge was not found among 
layer 5 corticofugal neuron pairs that do not receive thalamic inputs (Swadlow et al., 1998). On the other hand, spiking synchrony between barrel columns can be found if multiple whiskers are stimulated simultaneously or sequentially (Zhang and Alloway, 2004, 2006). Such results have been interpreted as evidence for thalamocortical inputs being the underlying organizer of spike synchrony in primary sensory areas (Swadlow and Lukatela, 1996). Also, it has been suggested that precisely correlated activity among thalamic neurons may strengthen the excitatory effects of thalamic input on cortical neurons through temporal summation (Alonso et al., 1996; Temereanca et al., 2008), which may be necessary because of the low efficacy of individual thalamocortical synapses (Bruno and Sakmann, 2006). The state of thalamic synchrony is predicted to change millisecond by millisecond as the whiskers are moved to make high-velocity contact with objects in the environment (Temereanca et al., 2008). Functionally, the summation of EPSPs may be especially effective in enhancing activity in the synaptic targets when peripheral stimulation is weak or nonoptimal (Kyriazi and Simons, 1993).

Septal cells with normally low correlation probably include neurons which have connections with structures outside the barrel field, and these areas may be less affected by deprivation. On the other hand, the septal cells that receive direct connections from nearest-neighbor barrels might develop high or intermediate synchrony with other types of septal cells during the course of maturation. It is possible that following deprivation when the ultralow lemniscal activity is insufficient to synchronize barrel cell activity, the septal cells connected to these deprived barrel neurons similarly fail to develop the expected synchrony. This feature of septal function is consistent with the idea that while the barrel neurons are strongly sensory, the septal neurons participate in more sensorimotor integrative functions (for review, see Alloway, 2008).

The fact that correlated discharge in barrel cortex is reduced during the response period after a stimulus and that following deprivation this difference does not develop in barrel-barrel and barrelseptum neuronal pairs (Fig. 5) hints at the importance of this reduction in correlated discharge for processing information in the barrels that is needed to guide behavior. This finding suggests that reduced correlated firing during the stimulus- evoked epoch also develops through the influence
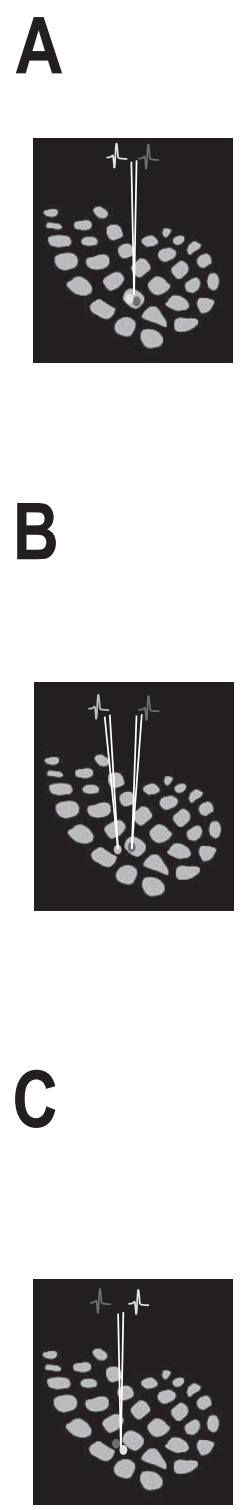
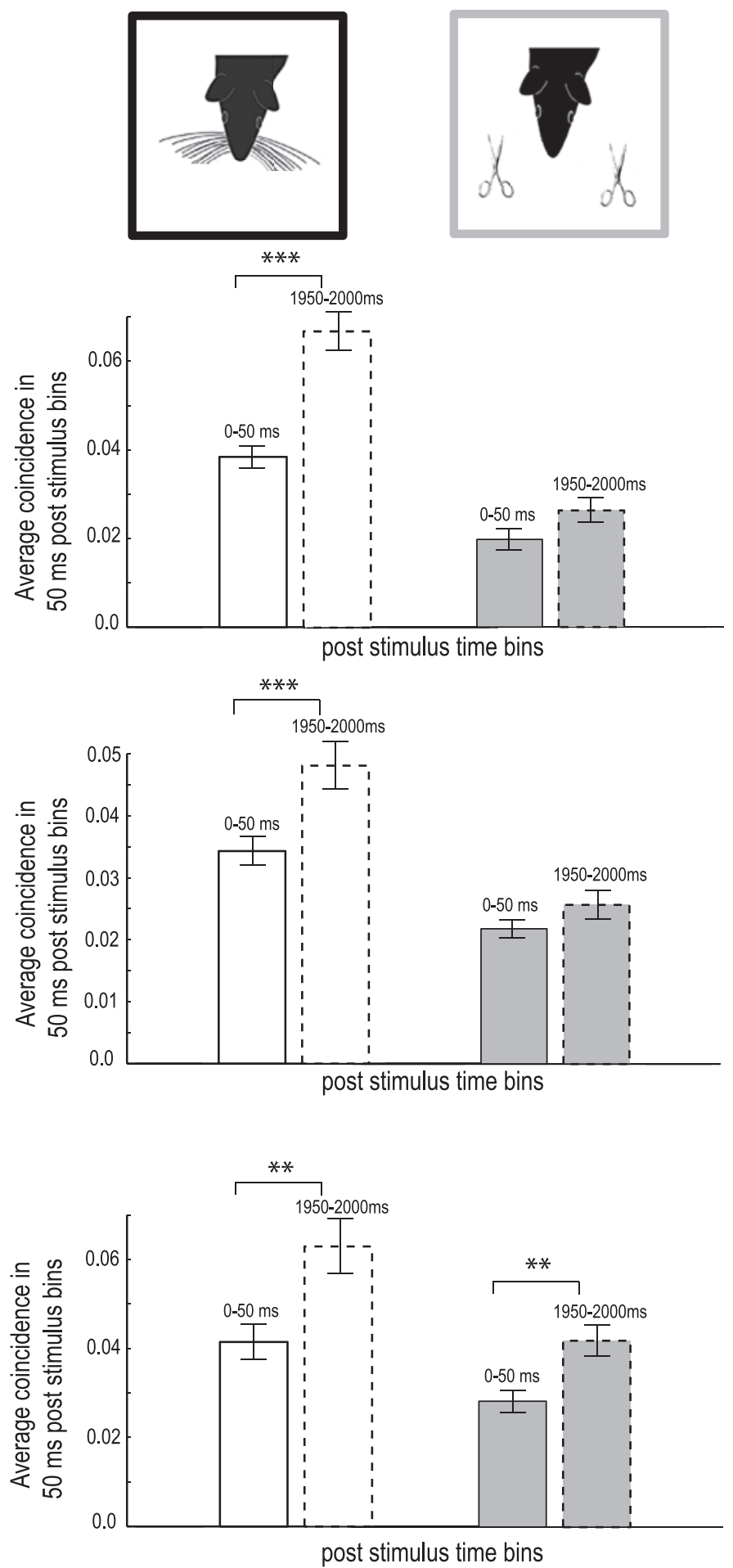

Figure 5. Comparison of the average coincidence in neuronal pairs in the "first" $50 \mathrm{~ms}$ after the stimulus (solid bars) and the "last" $50 \mathrm{~ms}$ (1950-2000 ms after stimulus, dashed bars) after best whisker stimulation in the three locations. Error bars represent SEM. The triple asterisks $\left(^{* * *}\right.$ ) and double asterisks ${ }^{* *}$ ) represent significance levels of $<0.001$ and $<0.01$, respectively. $A$, In barrel cells of CON animals (white bars), the "last" $50 \mathrm{~ms}$ (background activity epoch) shows significantly higher correlation than the "first" $50 \mathrm{~ms}$ (evoked response epoch) ( $p<0.001$; one-tailed $t$ test). This difference is eliminated after bilateral sensory deprivation (gray bars; $p>0.05$; one-tailed $t$ test). $\boldsymbol{B}$, Similar data when one cell is in a barrel and the other is in the septum. $\boldsymbol{C}$, When both cells are in a septal zone, the "first 50 , last 50 " difference is maintained after sensory deprivation, showing a resistance of septal cells to this effect of bilateral sensory deprivation.

of the active thalamocortical connections during normal rearing. In the population of deprived septal cells, this feature of correlated discharge continued to be present, possibly due to other 
A

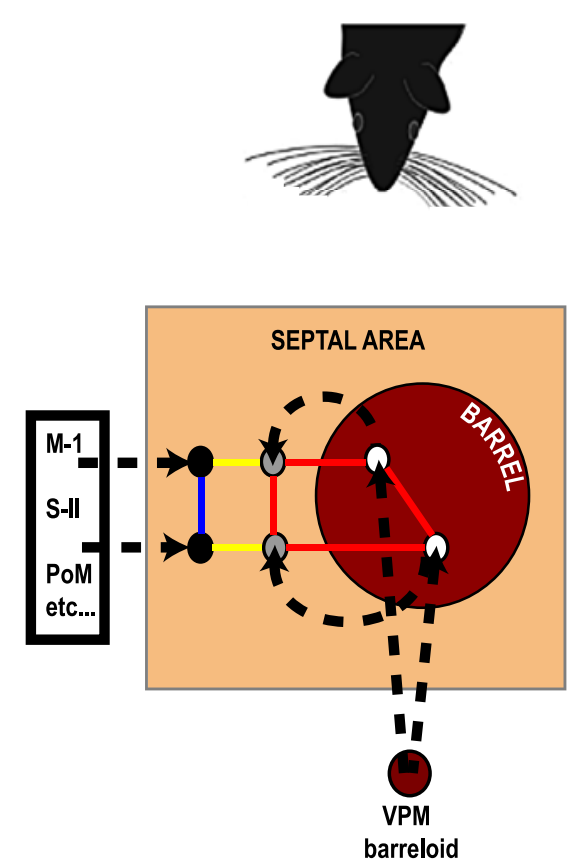

B
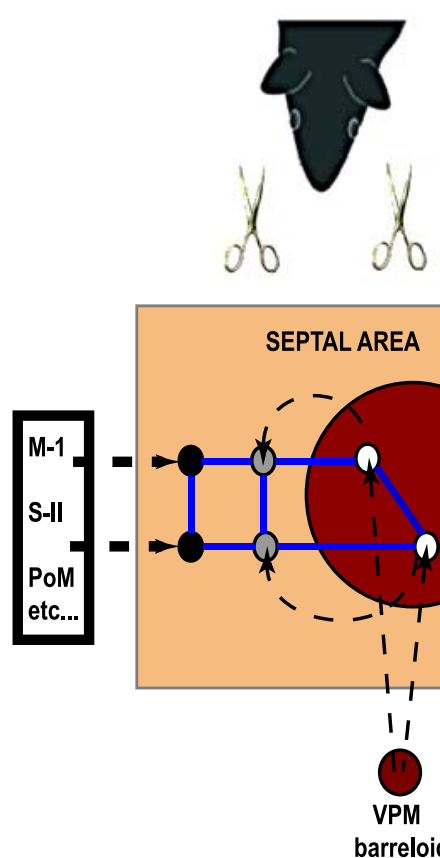

Figure 6. Model of the effect of sensory deprivation on the integrative properties of neurons in barrel cortex. Dashed lines with arrowheads represent connections and solid colored lines represent correlated activity. Thickness of the dashed line indicates the level of neuronal activity. Red, yellow, and blue solid lines represent high, intermediate, and low correlated activity. $\boldsymbol{A}$, During normal rearing, the lemniscal pathway through the relay nucleus of the thalamus (VPM) is very active and leads to strong correlated discharge (red lines) among barrel neurons (white circles) that receive VPM thalamic input. Barrel neurons, in turn, project to the septum around the barrel, developing strong correlated circuits (red lines) with a subset of septal neurons (gray circles) to which they are directly connected. Other neurons (black circles) in the septum are assumed to receive diverse inputs, many from outside the barrel cortex (box on the left), and these septal neurons develop only modest (blue line) correlated activity among themselves, and intermediate correlated activity (yellow lines) with the barrel-dominated septal neurons. $\boldsymbol{B}$, Sensory deprivation drastically reduces activity in the lemniscal pathway, and low activity blocks the development of correlated discharge between neurons in a barrel, and between barrel and a subset of septal neurons (blue lines). The non-barrel-dominated septal cells maintain their low level of correlated discharge even when the lemniscal inputs are low, presumably due to diverse inputs from outside the barrel cortex.

connections to the septum from outside the barrel field. The requirement for background spike synchrony in cortex coupled with the coding-based deficits in behavioral acuity lead to the hypothesis that the maturation of barrel network assemblies requires correlated discharge during development to generate normal processing in cortical networks. This model of normal and deprived cortical development is illustrated in Figure 6.

Figure $6 \mathrm{~A}$ illustrates a number of features of circuit maturation in barrel cortex that may be activity-sensitive during normal postnatal development. Activity imposes changes in cortical circuitry leading to the well established excitatory-inhibitory-excitatory response sequence well documented in barrel cortex (Simons and Carvell, 1989; Agmon and Connors, 1991). Within this response there is a strong theoretical case for almost all ( $\sim 91 \%)$ of the information about whisker location being conveyed by the first spike after the stimulus (Panzeri et al., 2001; Petersen et al., 2001). Following a brief burst of firing after a whisker contacts an object there is widespread inhibitory activity in the barrel, orchestrated by the synchronous discharge of nearly all inhibitory neurons in a barrel: cell activity within a barrel is thus effectively quenched after the initial high discharge rate (Swadlow et al., 1998). This level of correlated discharge, even at rest, is unusual and could maintain a high level of synaptic strength ("readiness") to respond as an ensemble to thalamic input volleys. Excitatory activity then rebounds at longer latency until the dis- charge in the barrel circuit returns to a low background level that is no longer directly tied to the stimulus, but continues to be correlated among neurons in each barrel (present results). Bilateral sensory deprivation has a negative impact on each of these activity-based events. First, the response of both excitatory and inhibitory neurons to a thalamic volley in deprived barrel cortex is significantly weaker when individual cells respond to single test stimuli (fewer spikes per stimulus), compared with that elicited in normally reared animals. Further, deprivation disrupts the timing of the first spike after stimulus and the high probability of short (7-10 ms)-latency discharge following repeated stimulations (Popescu and Ebner, unpublished observations) (supplemental Fig. 1, available at www.jneurosci.org as supplemental material). Finally, the barrel neurons fail to develop the characteristic "synchrony at rest" found in numerous studies of normal barrel cortex (Swadlow et al., 1998; Peterson et al., 2001; Zhang and Alloway, 2006; present results). The net result is a sensory-deprived cortex that looks anatomically quite normal, but that cannot process sensory information with normal precision. Importantly, this synchronous spontaneous discharge is also seen in awake preparations, and it is not abolished by anesthesia (Swadlow et al., 1998). Awake behaving preparations will be necessary to determine whether synchronized activity is increased by attention to task as it is in the secondary somatic sensory area of primates (Roy et al., 2007).

Despite the observation that spike synchrony is severely reduced following early sensory deprivation, it remains to be directly tested whether or not spike synchrony is a sufficient or even a necessary mechanism for development of normal sensory processing. It is possible that changes in synchrony will be correlated with the rapid fluctuations in plasticity that occur in barrel cortex (Erchova and Diamond, 2004). If so, low levels of correlation also could be a cause of reduced plasticity in barrel cortex following early sensory deprivation. However, evidence from awake behaving animals would be necessary to conclusively analyze the behavioral consequences of the loss in neuronal synchrony caused by sensory deprivation.

\section{References}

Abbott LF, Dayan P (1999) The effect of correlated variability on the accuracy of a population code. Neural Comput 11:91-101.

Aertsen AM, Gerstein GL, Habib MK, Palm G (1989) Dynamics of neuronal firing correlation: modulation of "effective connectivity." J Neurophysiol 61:900-917.

Agmon A, Connors BW (1991) Thalamocortical responses of mouse somatosensory (barrel) cortex in vitro. Neuroscience 41:365-379.

Alloway KD (2008) Information processing streams in rodent barrel cortex: the differential functions of barrel and septal circuits. Cereb Cortex 18:979-989.

Alonso JM, Usrey WM, Reid RC (1996) Precisely correlated firing in cells of the lateral geniculate nucleus. Nature 383:815-819.

Brody CD (1999) Correlations without synchrony. Neural Comput 11:1537-1551. 
Brosch M, Schreiner CE (1999) Correlations between neural discharges are related to receptive field properties in cat primary auditory cortex. Eur J Neurosci 11:3517-3530.

Bruno RM, Sakmann B (2006) Cortex is driven by weak but synchronously active thalamocortical synapses. Science 312:1622-1627.

Carvell GE, Simons DJ (1996) Abnormal tactile experience early in life disrupts active touch. J Neurosci 16:2750-2757.

Eggermont JJ (2001) Between sound and perception: reviewing the search for a neural code. Hear Res 157:1-42.

Eggermont JJ (2006) Properties of correlated neural activity clusters in cat auditory cortex resemble those of neural assemblies. J Neurophysiol 96:746-764.

Eggermont JJ (2007) Correlated neural activity as the driving force for functional changes in auditory cortex. Hear Res 229:69-80.

Engel AK, König P, Singer W (1991) Direct physiological evidence for scene segmentation by temporal coding. Proc Natl Acad Sci USA 88:9136-9140.

Erchova IA, Diamond ME (2004) Rapid fluctuations in rat barrel cortex plasticity. J Neurosci 24:5931-5941.

Friedberg MH, Lee SM, Ebner FF (1999) Modulation of receptive field properties of thalamic somatosensory neurons by the depth of anesthesia. J Neurophysiol 81:2243-2252.

Gerstein GL, Bedenbaugh P, Aertsen MH (1989) Neuronal assemblies. IEEE Trans Biomed Eng 36:4-14.

Hsiao SS, O'Shaughnessy DM, Johnson KO (1993) Effects of selective attention on spatial form processing in monkey primary and secondary somatosensory cortex. J Neurophysiol 70:444-447.

Kim U, Ebner FF (1999) Barrels and septa: separate circuits in rat barrels field cortex. J Comp Neurol 408:489-505.

Kyriazi HT, Simons DJ (1993) Thalamocortical response transformations in simulated whisker barrels. J Neurosci 13:1601-1615.

Lee SH, Land PW, Simons DJ (2007) Layer- and cell-type-specific effects of neonatal whisker-trimming in adult rat barrel cortex. J Neurophysiol 97:4380-4385.

Li L, Rema V, Ebner FF (2005) Chronic suppression of activity in barrel field cortex downregulates sensory responses in contralateral barrel field cortex. J Neurophysiol 94:3342-3356.

Miller R (1996) Neural assemblies and laminar interactions in the cerebral cortex. Biol Cybern 75:253-261.

Mitchell DE (1988) The extent of visual recovery from early monocular or binocular visual deprivation in kittens. J Physiol 395:639-660.

Panzeri S, Petersen RS, Schultz SR, Lebedev M, Diamond ME (2001) The role of spike timing in the coding of stimulus location in rat somatosensory cortex. Neuron 29:769-777.

Petersen RS, Diamond ME (2000) Spatial-temporal distribution of whisker-evoked activity in rat somatosensory cortex and the coding of stimulus location. J Neurosci 20:6135-6143.

Petersen RS, Panzeri S, Diamond ME (2001) Population coding of stimulus location in rat somatosensory cortex. Neuron 32:503-514.

Rema V, Armstrong-James M, Ebner FF (2003) Experience-dependent plasticity is impaired in adult rat barrel cortex after whiskers are unused in early postnatal life. J Neurosci 23:358-366.

Rolls ET, Franco L, Aggelopoulos NC, Reece S (2003) An information theoretic approach to the contributions of the firing rates and the correlations between the firing of neurons. J Neurophysiol 89:2810-2822.

Roy A, Steinmetz PN, Hsiao SS, Johnson KO, Niebur E (2007) Synchrony: a neural correlate of somatosensory attention. J Neurophysiol 98:1645-1661.

Sceniak MP, Maciver MB (2006) Cellular actions of urethane on rat visual cortical neurons in vitro. J Neurophysiol 95:3865-3874.

Shepherd GM, Pologruto TA, Svoboda K (2003) Circuit analysis of experience-dependent plasticity in the developing rat barrel cortex. Neuron 38:277-289.

Shoykhet M, Land PW, Simons DJ (2005) Whisker trimming begun at birth or on postnatal day 12 affects excitatory and inhibitory receptive fields of layer IV barrel neurons. J Neurophysiol 94:3987-3995.

Simons DJ, Carvell GE (1989) Thalamocortical response transformation in the rat vibrissa/barrel system. J Neurophysiol 61:311-330.

Simons DJ, Land PW (1987) Early experience of tactile stimulation influences organization of somatic sensory cortex. Nature 326:694-697.

Swadlow HA, Lukatela K (1996) Cross-correlation and microstimulation: complementary tools in the extracellular analysis of synaptic interactions. J Neurosci Methods 64:219-225.

Swadlow HA, Beloozerova IN, Sirota MG (1998) Sharp, local synchrony among putative feed-forward inhibitory interneurons of rabbit somatosensory cortex. J Neurophysiol 79:567-582.

Temereanca S, Brown EN, Simons DJ (2008) Rapid changes in thalamic firing synchrony during repetitive whisker stimulation. J Neurosci 28:11153-11164.

Ventura V, Cai C, Kass RE (2005) Trial-to-trial variability and its effect on time-varying dependency between two neurons. J Neurophysiol 94:2928-2939.

Wiesel TN, Hubel DH (1965) Extent of recovery from the effects of visual deprivation in kittens. J Neurophysiol 28:1060-1072.

Zhang M, Alloway KD (2004) Stimulus-induced intercolumnar synchronization of neuronal activity in rat barrel cortex: a laminar analysis. J Neurophysiol 92:1464-1478.

Zhang M, Alloway KD (2006) Intercolumnar synchronization of neuronal activity in rat barrel cortex during patterned airjet stimulation: a laminar analysis. Exp Brain Res 169:311-325. 